\title{
Families of SOA Migration
}

\author{
Maryam Razavian^ and Patricia Lago \\ Department of Computer Science, VU University Amsterdam, The Netherlands \\ $\{$ m.razavian, p.lago\}@few.vu.nl
}

\begin{abstract}
Migration of legacy systems to SOA constitutes a key challenge of service-oriented system engineering. Despite the many works around such migration, there is still little conceptual characterization on what SOA migration entails. To solve this problem, we conducted a systematic literature review that extracts main categories of SOA migration, called SOA migration families, from the approaches proposed in the research community. Based on the results of the systematic review, we describe eight distinct families along with their characteristics and goals.
\end{abstract}

\section{Introduction}

One of the key promises of service oriented paradigm is facilitating reuse of enterprise assets in legacy systems. Migration of legacy systems to service-based systems enables achieving advantages offered by SOA while still reusing the embedded capabilities in the legacy systems. Various studies present an approach for such migration. These studies mainly differ in the way they provide solutions for two challenging problems of what can be migrated (i.e. the legacy elements) and how the migration is performed (i.e. the migration process). Such differences can hinder achieving a general understanding of 'what SOA migration entails' and therefore making it difficult to determine how to migrate.

To obtain such understanding, we conducted a systematic literature review that extracts main migration categories existing in the field. Due to its methodological rigor, we chose systematic review as our research method in aggregating existing SOA migration approaches. Furthermore, the strength of systematic reviews in minimizing the bias in the review process enhances the extraction of sound and meaningful migration categories. By devising a coding procedure, we analyzed the studies and extracted eight distinct categories. Using a holistic conceptual framework that reflects distinct conceptual elements involved in the migration process, SOA migration families are typified in a unified manner. As such, these families act as a frame of reference for SOA migration which brings order and enhances understanding in how such migration can be carried out. Accordingly, this frame of reference increases awareness of the ways in which a legacy system can be migrated to SOA.

\footnotetext{
* This research has been partially sponsored by the Dutch Jacquard program on Software Engineering Research via contract 638.001.206 SAPIENSA: Service-enAbling PreexIsting ENterprISe Assets; and the European Community's FP7/2007-2013 under grant agreement 215483 (S-Cube).
} 


\section{SOA Migration Families}

By applying the pre-defined protocol of the systematic review, we identified 39 primary studies. Using qualitative analysis techniques, each of the primary studies was mapped to a framework represented in Fig. 1 called SOA-MF. By considering similar SOA-MF coverage patterns, out of 39 different mappings eight distinct families of SOA migration approaches were extracted. Fig. 1.III illustrates the schematic form of distinguished mappings that are dedicated to each family. As an example, F4.b is a schematic form of the mapping shown in Fig 1.II. At first glance, a SOA migration family represents a set of approaches with graphically similar mappings on SOA-MF. Despite their simplicity, the mappings reflect the following information about the migration process: to what extent the reverse engineering, transformation and forward engineering occur, what activities are carried out, what artifacts are used or produced, and what abstraction levels are covered. By positioning a migration approach on these families, insight in the following aspects can be achieved: what is migrated, how the migration is carried out, what is the main objective of migration, and finally, what are the available solutions. More details are outlined at http://www.few.vu.nl/ mrazavi/SOAMigrationAppendix.pdf.
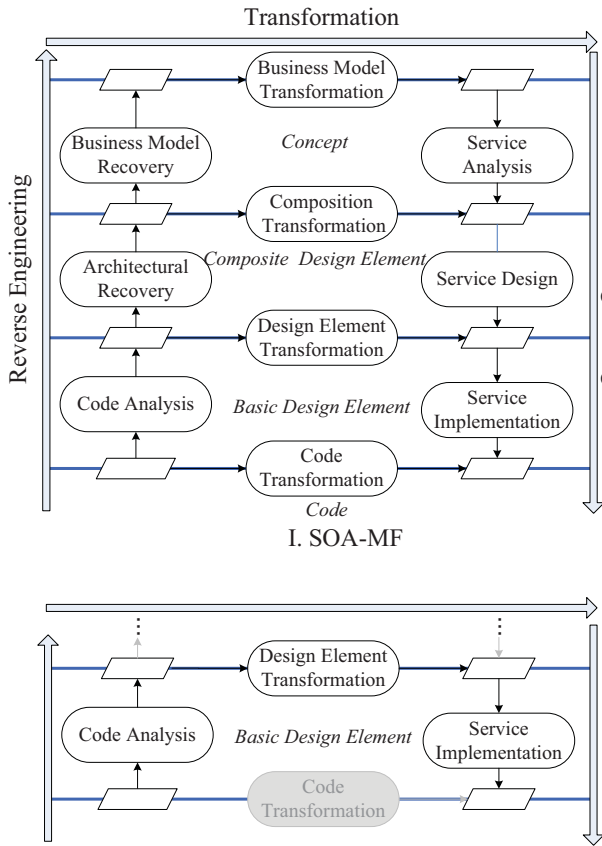

II. Mapping of F4(b) on SOA-MF

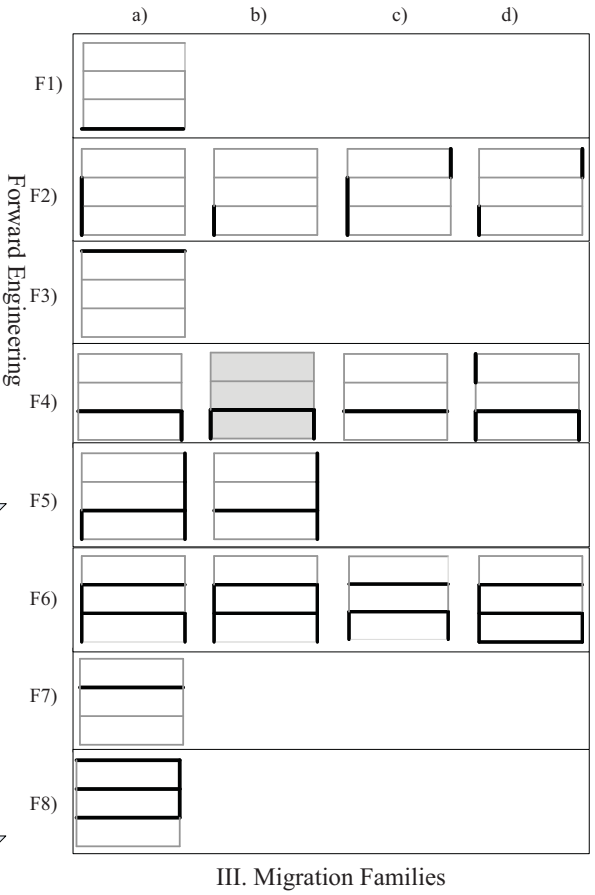

Fig. 1. SOA Migration Families 